\title{
Modeling DM and ICM in simulated galaxy clusters
}

\author{
E. Rasia ${ }^{1}$, G. Tormen ${ }^{1}$ and L. Moscardini ${ }^{2}$ \\ ${ }^{1}$ Dipartimento di Astronomia, Università di Padova, vicolo dell'Osservatorio 2, I-3512, Padova, \\ Italy email: rasia@pd.astro.it \\ ${ }^{2}$ Dipartimento di Astronomia, Università di Bologna, via Ranzani 1, I-40127, Bologna, Italy
}

\begin{abstract}
Using the results of an extended set of high-resolution non-radiative hydrodynamic simulations of galaxy clusters we obtain simple analytic formulae for the dark matter and hot gas distribution, in the spherical approximation.
\end{abstract}

\section{Introduction}

Galaxy clusters are the largest virialized systems we observe in the universe today. Any comparison with observations in the optical or via gravitational lensing requires modeling at least the dark matter (DM) component of galaxy clusters, while X-ray or millimetric observations (e.g. the Sunyaev-Zel'dovich effect) require knowledge of the internal structure of the Intra Cluster Medium (ICM). Both aspects can in principle be studied by means of numerical simulations.

In this paper we summarize some of our results: an extended discussion can be found in Rasia et al. (2004).

Numerical Simulations. The sample includes 17 high-resolution clusters, simulated with the hydro-N-body code GADGET (Springel et al. 2001). Their virial masses range between $3.1 \times 10^{14} h^{-1} M_{\odot}$ and $1.7 \times 10^{15} h^{-1} M_{\odot}$ and virial radii, $R_{\mathrm{v}}$, between 1.4 and $2.5 h^{-1}$ Mpc. Our analysis is restricted to the $z=0$ outputs. Thanks to the random criterion used to select the re-simulated haloes, the modeling we are doing will be representative of an average cluster, in an average environment and dynamical configuration.

\section{Modeling dark matter profiles}

The underlying idea of this work is to build a self-consistent model for the radial profiles describing the dynamical properties of galaxy clusters. For each cluster we compute the radial profile of the interested quantity, then we average the profiles and fit this using at most double power-law analytic fits. We decided to consider all the profiles presented in this work only at radii larger than $\log x=-1.85$, where $x \equiv r / R_{\mathrm{v}}$. Within this radius our clusters contain on average $\approx 800$ dark matter particles. In all plots the solid line is the average profile, the shaded region represents the error on the mean and the vertical dashed line is the inner-radius limit of our model.

Density structure. A model for the dark matter density profile is straightforward to obtain by combining our coarse-grained phase-space density model, $\bar{f}(x) \equiv \rho(x) / \sigma_{r}^{3}(x) \propto$ $x^{-1.875}$, with the radial velocity dispersion profile $\tilde{\sigma}_{r}(x)=0.67 x^{0.3}\left(x+x_{p}\right)^{-0.48}$, with $x_{p}=10^{-1.16}$ and solving for the density. We easily get the following approximate fit for 

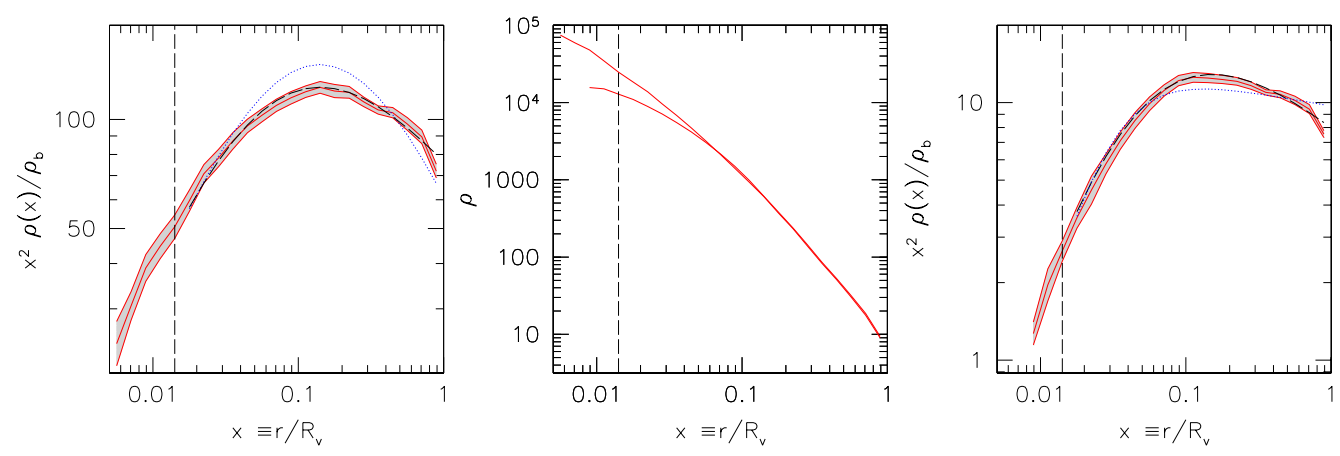

Figure 1. Density profiles. Left (Right) panel: average dark matter (gas) profile $\rho(x) x^{2}$. The dashed line is our fit, while the dotted lines is the NFW model ( $\beta$-model). Central panel: gas (solid) and dark matter (dashed) density profile normalized so that they match at the virial radius.

the dark matter spatial density distribution:

$$
\tilde{\rho}(x)=\frac{\rho_{0}}{x\left(x+x_{p}\right)^{1.5}} ; \rho_{0}=\frac{\left(1-f_{\mathrm{b}}\right) \Delta_{\mathrm{v}}}{6\left[\left(1+2 x_{p}\right) /\left(1+x_{p}\right)^{1 / 2}-2 x_{p}^{1 / 2}\right]},
$$

where $\Delta_{\mathrm{v}}$ is the virial over-density specified by the cosmological model and $f_{\mathrm{b}}=0.097$ is the average baryonic fraction.

The mean density profile, averaged over the whole sample of simulated clusters, is shown in Fig. 1, our model (eq. 2.1) is represented by the dashed line, while with the dotted line we represent the NFW model (Navarro, Frenk \& White 1997), with a concentration parameter equal to $c=1 / x_{s}=6.3$.

Mass estimates. Given the previous relations, the cluster mass inside any given radius can be obtained in different ways: by integrating our density profile (eq. 2.1), or by integrating the NFW profile, or through the Jeans equation. We found that the Jeans equation is a proper description of the mass, being the error always smaller than 10 per cent and that the mass derived by integrating our density profile is in very good agreement with the actual mass.

\section{Modeling gas profiles}

Density structure. In analogy to what has been done in the previous section for the dark matter profiles, in this section we will apply a similar analysis to the radial profiles of the hot gas component. Let us start from the density. In the central panel of Fig. 1 we compare the profiles of gas and dark matter, normalized so that they match at the virial radius.

The profiles of gas and dark matter are roughly self-similar for $x>0.06$, while the gas profile becomes flatter in the internal region. The self-similarity of the DM and gas profiles in the outer part of the cluster suggested us to fit the gas density profile with the same functional form used for the dark matter, with a different concentration value, relaxing the constraint on the inner slope and assuming of course a different normalization 


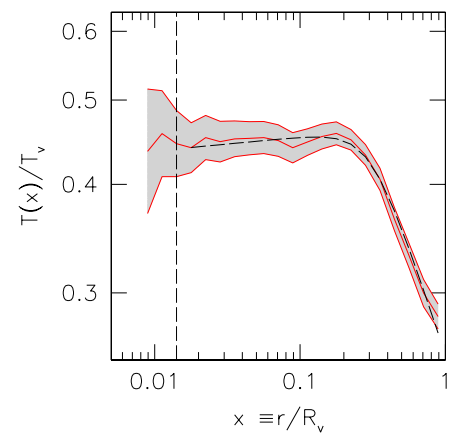

Figure 2. Average gas temperature profile for our model (dashed curve).

based on the average baryon fraction:

$$
\tilde{\rho}(x) \equiv \frac{\rho(x)}{\rho_{\mathrm{b}}}=\frac{\rho_{0}}{\left(x+x_{p}\right)^{2.5}}, \rho_{0}=\frac{f_{\mathrm{b}} \Delta_{\mathrm{v}}}{3} \times\left[\frac{16 x_{p}^{3} / 3+40 x_{p}^{2} / 3+10 x_{p}+2}{\left(x_{p}+1\right)^{2.5}}-\frac{16 x_{p}^{1 / 2}}{3}\right]^{-1}
$$

where $x_{p}=0.04$;

In the right panel of Fig. 1 we compare the average profile for the gas density obtained from our simulated clusters (solid curve) with the proposed fit (dashed curve) and with the $\beta$-model (Cavaliere \& Fusco-Femiano 1976, 1978)(dotted line).

Temperature structure. Observations suggest the presence of a central isothermal region followed by a smooth decline towards the outskirts and there are strong indications that the value of the temperature at the virial radius is approximately a factor of 2 smaller than at the center.

In Fig. 2 we show the average temperature profile normalized by the estimate of the virial temperature, $T_{\mathrm{v}} \equiv\left(G \mu m_{p} M_{\mathrm{v}}\right) /\left(k_{\mathrm{b}} R_{\mathrm{v}}\right)$. In this relation $G$ is the gravity constant, $\mu=0.59$ is the mean molecular weight, $m_{p}$ is the proton mass and $k_{\mathrm{b}}$ is the Boltzmann constant.

The sudden temperature drop at $x \approx 0.2$ cannot be fit by a double power-law as those used so far. Instead, we model this sharp transition by using the following expression: $T(x) / T_{\mathrm{v}}=\left(0.255 x^{0.016}\right) /\left[\left(x^{4}+x_{p}^{4}\right)^{0.13}\right]$, where $x_{p}=10^{-0.51}$.

Mass estimates. A typical mass estimator using ICM as a tracer and assuming a spherical and static gravitational potential is the equation of the hydrostatic equilibrium. However, the hypotheses underlying this equation are too restrictive for at least two reasons. First, our simulations show that the mean velocity anisotropy $\beta(x)$ is not zero, second the residual bulk motions of the gas must be taken into account.

A more proper mass estimate, including the radial velocity dispersion and the velocity anisotropy, is given by:

$$
M^{E}(<x)=-x M_{v} \tilde{T}(x)\left[\frac{d \ln \tilde{\rho}(x)}{d \ln x}+\frac{d \ln \tilde{T}(x)}{d \ln x}\right]+x M_{v} \tilde{\sigma}_{r}^{2}(x)\left[\frac{d \ln \tilde{\rho}(x)}{d \ln x}+\frac{d \ln \tilde{\sigma}_{r}^{2}(x)}{d \ln x}+2 \tilde{\beta}(x)\right] .
$$

We found that the $\beta$-model underestimates the true cluster mass at most radii, whereas using our density profile improves the estimate at all radii and reduces the error at the virial radius to 15 per cent. The complete model is the most accurate at all radii, with an error of 5 to 10 per cent at $x>0.2$. 


\section{References}

Cavaliere A., Fusco-Femiano R. 1976, A\&\&A 49, 137

Cavaliere A., Fusco-Femiano R. 1978, A\&A 70, 677

Navarro J.F., Frenk C.S., White S.D.M. 1997 ApJ 490493

Rasia E., Tormen G., Moscardini L. 2004, MNRAS, 351, 237

Springel V., Yoshida N., White S.D.M. 2001 NewA 679 